\title{
Short-Term Progression of Diabetic Hard Exudates Monitored with High-Resolution Camera
}

\author{
Prisca Loganadane Bernard Delbosc Maher Saleh \\ Department of Ophthalmology, Besançon University Hospital, Besançon, France
}

\section{Keywords}

High-resolution adaptive optics camera - Diabetic macular edema $\cdot$ Hard exudates

\begin{abstract}
Purpose: To assess the progression of diabetic hard exudates over an 8-week period, using a high-resolution adaptive optics camera. Design: Prospective observational study. Methods: Five eyes of three patients presenting diabetic maculopathy with hard exudates were studied. An area of clinically visible exudates was imaged sequentially using SDOCT and high-resolution flood illumination adaptive optics for 2 months, on a weekly basis. A time-lapse video was obtained for each eye studied. Changes in terms of surface, number of free elements (foci), and central macular thickness were recorded. Results: Short-term modifications in terms of disposition, size, and number of exudates were observed. Two patterns of progression were identified: two eyes showed exudate dislocation concomitant with the regression of the underlying macular edema, with hard exudates being progressively replaced by a multitude of smaller foci. These exudates were labeled resorption exudates. In three other eyes with persistent diabetic macular edema, foci aggregated into larger exudates. Conclusion: Diabetic hard exudate changes occurred over a short period of time but were not assessable clinically. Adaptive optics was able to document these subtle changes precisely. Further studies
\end{abstract}

\section{KARGER}

(๑) 2018 S. Karger AG, Basel

E-Mail karger@karger.com

www.karger.com/ore using this imaging modality may improve our understanding of the natural history of exudates and eventually help assess the efficacy of the various treatments available such as lipid-lowering drugs and anti-VEGFs injections.

(c) 2018 S. Karger AG, Basel

\section{Introduction}

Diabetic macular edema (DME) is a leading cause of visual loss in diabetic patients [1]. DME results from a breakdown of the blood-retina barrier of intraretinal vessels, leading to an enhanced lipid and protein exudation clinically labeled hard exudates (HEs) [2]. HEs are frequently associated with a high level of serum lipids $[1,3]$ and can contribute to visual loss when they are located in the foveal area $[1,4-6]$. For years, retinography was the gold standard in assessing HE size on a set of standard stereoscopic color photographs as proposed by the Early Treatment Diabetic Retinopathy Study [1, 7, 8]. More recently, spectral-domain optical coherence tomography (SD-OCT) has improved our understanding of the natural history of HEs. Bolz et al. $[9,10]$ reported the existence of foci, deposits located within the walls of intraretinal microaneurysms that are scattered throughout all retinal layers, forming confluent plaques in the outer plexiform layer [11]. Various treatments including lipid-lowering agents [1], photocoagulation [4], surgical excision [12], 
Table 1. Demographic and clinical characteristics of the patients studied

\begin{tabular}{|c|c|c|c|c|c|c|c|c|c|}
\hline Patient & Eye & $\begin{array}{l}\text { Age, } \\
\text { years }\end{array}$ & Gender & $\begin{array}{l}\text { Hbalc, } \\
\%\end{array}$ & $\begin{array}{l}\text { Blood } \\
\text { pressure, } \\
\text { mm Hg }\end{array}$ & $\begin{array}{l}\text { Antihyper- } \\
\text { tensive } \\
\text { drugs }\end{array}$ & Statins & $\begin{array}{l}\text { CFT at } \\
\text { baseline, } \\
\mu \mathrm{m}\end{array}$ & $\begin{array}{l}\text { Final } \\
\text { CFT, } \\
\mu \mathrm{m}\end{array}$ \\
\hline 1 & $\begin{array}{l}1 \\
2\end{array}$ & 49 & Male & 11.1 & $140 / 90$ & Yes & Yes & $\begin{array}{l}354 \\
319\end{array}$ & $\begin{array}{l}319 \\
272\end{array}$ \\
\hline 2 & $\begin{array}{l}3 \\
4\end{array}$ & 46 & Male & 7.6 & $150 / 80$ & No & Yes & $\begin{array}{l}345 \\
363\end{array}$ & $\begin{array}{l}340 \\
376\end{array}$ \\
\hline 3 & 5 & 43 & Male & 11.7 & $140 / 85$ & Yes & No & 410 & 398 \\
\hline
\end{tabular}

intravitreal injections of steroids [13], or more recently, antiangiogenic agents $[14,15]$ have been proposed to promote their regression. Previous assessment using SDOCT and/or retinography have shown HE regression after several months $[4,11]$, mostly by macrophage phagocytosis. Recently, a new imaging modality, adaptive optics (AO), based on the correction of the optical aberrations of the eye, has contributed new insight into the retinal imaging field by providing real-time images of the retinal structure a few microns in size such as photoreceptor cells, the vessel wall, or pigment migration [16-19]. Yamaguchi et al. [20] imaged HEs using an AO scanning laser ophthalmoscope (AOSLO) and classified them into round or irregular shape, the first category eventually transforming into the second. In this study, we assessed the change in HEs using a flood illumination AO device. For this purpose, HEs were imaged sequentially over an 8 -week period and their progression was compared to that of the underlying DME.

\section{Methods}

An institutional observational prospective study was conducted in the Ophthalmology Department of the University Hospital of Besançon (France). The local ethics committee of the Besançon University Hospital approved the protocol and it adhered to the tenets of the Declaration of Helsinki. The nature of the study and its possible consequences were explained to study candidates, after which written informed consent was obtained from all participants.

\section{Participants}

Patients presenting diabetic maculopathy with HE seen on indirect ophthalmoscopy were studied. Five eyes of three men aged from 43 to 67 years were studied. The baseline characteristics of the patients studied are summarized in Table 1. All patients underwent a comprehensive ophthalmologic examination including best corrected visual acuity measurement. Ocular axial length was measured using an IOL Master ${ }^{\circledR} 500$ (Carl Zeiss Meditec, Dublin,
CA, USA). Multimodal imaging of an area of clinically visible exudates in the parafoveal area was conducted, encompassing retinography (Kowa Nonmyd 7, Kowa Ltd, Berkshire, UK), spectral-domain OCT (Heidelberg Engineering, Heidelberg, Germany), and AO imaging (RTX1, Imagine Eyes, Orsay, France). Images were acquired on a weekly basis over 2 months. A time-lapse video of the region of interest imaged using $\mathrm{AO}$ was obtained for each eye studied.

Exclusion criteria for this study aimed to obtain the best quality of retinal images for analysis. They included any condition impairing the media clarity, high myopia, astigmatism known to produce high-order aberrations, patients presenting a significant tear film disorder, insufficient pharmacologically induced mydriasis (pupil diameter $<5 \mathrm{~mm}$ ), uncontrolled movements of the eyes or the head, and poor fixation. Also excluded were patients presenting hemorrhage and pigment changes masking the retinal structures.

\section{Adaptive Optics Imaging}

Eyes were imaged using a flood illumination $\mathrm{AO}$ camera based on a noncoherent flood-illuminated design with an 850-nm central illumination wavelength allowing a lateral resolution between 2 and $4 \mu \mathrm{m}$. Its low-noise CCD camera has a pixel resolution of 1.6 $\mu \mathrm{m}$ and a frame rate of $9.5 \mathrm{fps}$. Imaging sessions were conducted after the pupils were dilated with two drops each of $0.5 \%$ tropicamide. The examiner determined a macular area of clinically visible exudates within a $4 \times 4$ degree square. The imaging depth was chosen within the range of $0-800 \mu \mathrm{m}$ in which the HE images appeared to be the sharpest.

\section{Image Processing and Time-Lapse Sequence}

Each series of 40 images acquired by the AO camera was processed using software programs provided by the system's manufacturer (CK v0.1 and AO detect v0.1, Imagine Eyes, France). These images were registered using a cross-correlation method and averaged to produce a final image with an improved signal-tonoise ratio. The raw images that showed artifacts due to eye blinking and saccades were automatically eliminated before averaging. On the AO image, HEs appeared as hyperreflective aggregates with various shapes at least $50 \mu \mathrm{m}$ wide. They were made of an aggregation of round elements of nearly the same size $(30 \mu \mathrm{m})$. Isolated 30 to $50 \mu \mathrm{m}$ elements were defined as foci. Foci were round, mainly hyperreflective with a darker shady portion. At higher magnification, their aspect was grained with hyperreflective dots on a dark- 

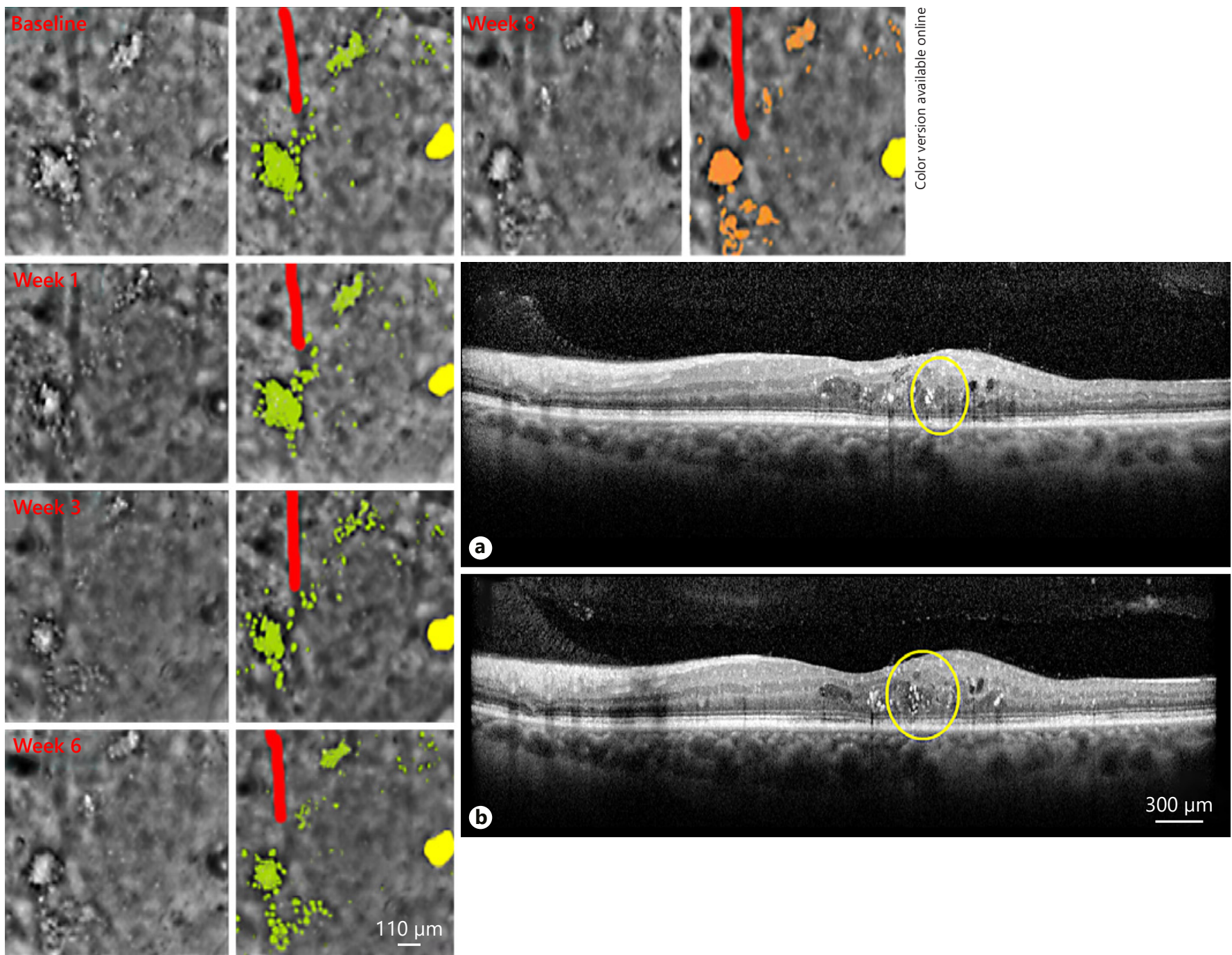

Fig. 1. Example of hard exudate (HE) regression (patient 1). Sequential imaging is presented between baseline and week 8 . Raw image and the colorized image are presented for each imaging session. Vascular landmarks are represented by normal retinal vasculature (red) and microaneurysm (yellow). Green, HEs and free

er background. Microfoci were elements smaller than $30 \mu \mathrm{m}$ (see online suppl. Material 1; for all online suppl. material, see www. karger.com/doi/10.1159/000493858). Their shape and size were more heterogeneous than the foci. However, their inner part displayed the same appearance as foci.

The number of imaging sessions ranged from seven to eight, with a mean interval between imaging sessions lasting 7 days and a 2-month follow-up.

To obtain time-lapse videos, averaged images of a given site were aligned and gif animations were constructed using i2k Align Retina software (DualAlign, LLC). The images were superimposed according to anatomical landmarks, mainly retinal vessels and microaneurysms. To evaluate the progression of HEs, the HE surface was measured by two independent graders using ImageJ software

Short-Term Progression of Diabetic HEs Monitored with High-Resolution Camera foci. A progressive dislocation of the HE is observed with a progressive replacement by a multitude of foci. $\mathbf{a}, \mathbf{b}$ Cross-sectional OCT scan at baseline (a) and at week $8(\mathbf{b})$. The area imaged is encircled.

(National Institutes of Health, Bethesda, MD, USA) and the free foci were counted in a 1-degree circle from the center of the HE studied. The data presented are the average of the two measurements.

\section{Results}

Changes in size and location of exudates together with the variation of free elements around the exudates were observed during the study period. Two patterns of progression were identified: 


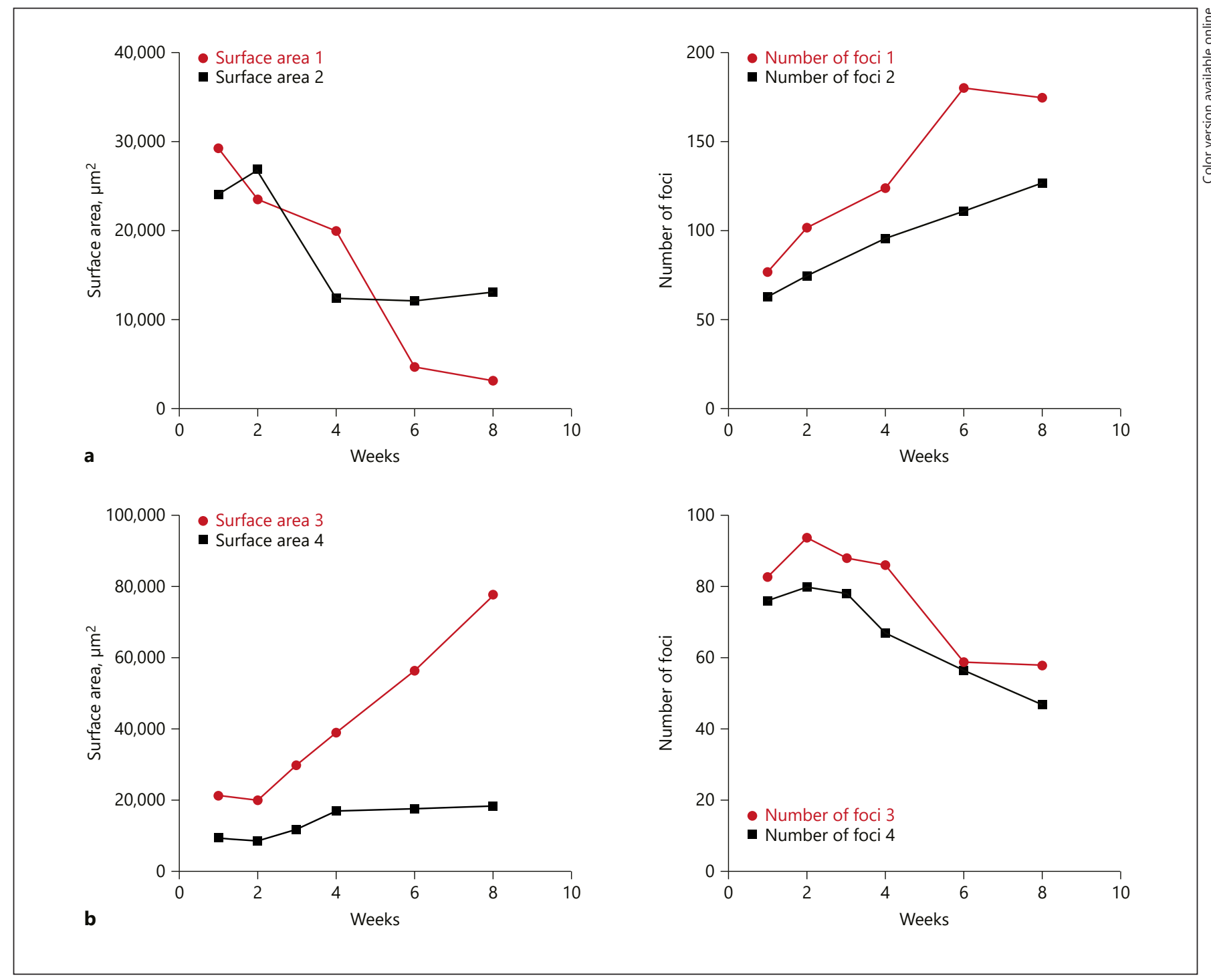

Fig. 2. Example of two different progression patterns. a Hard exudate (HE) resorption. Progression of HE in eye 1 (red) and eye 2 (black) showing a decrease in surface area, while the number of free foci in the neighborhood increased. $\mathbf{b}$ Exudate formation pat- tern in eye 3 (red) and eye 4 (black). The surface of the exudates increased in the region studied and the number of foci decreased as foci aggregated.
- HE regression: two eyes showed exudate dislocation concomitant with the regression of the underlying macular edema with HEs being progressively replaced by a multitude of smaller foci (Fig. 1 and 3). In the first case, the surface area of the resorption exudates at baseline was $29.403 \mu \mathrm{m}^{2}$ and $3,206 \mu \mathrm{m}^{2}$ at 2 months. At the same time, the number of foci increased from 77 to 175 at 2 months. The CRT decreased by $47 \mu \mathrm{m}$ (Fig. 2a). In the second case, the exudate surface decreased from $24.194 \mu \mathrm{m}^{2}$ to $13.108 \mu \mathrm{m}^{2}$ and the number of foci in the studied area rose from 63 to 127 elements (Fig. 2a).
- The other pattern observed in three eyes was an increase in HE size. In two eyes, the foci tended to progressively aggregate until forming a larger deposit while the number of free foci decreased. Cross-sectional OCT scans confirmed the predominance of the HEs in the outer plexiform layer (see online suppl. Material 2 and Fig. 3). The baseline surface in the first case was $21.500 \mu \mathrm{m}^{2}$ and increased to $78.129 \mu \mathrm{m}^{2}$ at 2 months. At the same time, the macular edema increased by $13 \mu \mathrm{m}$. The number of foci decreased from 83 to 58 at 2 months. In the second case, the surface 

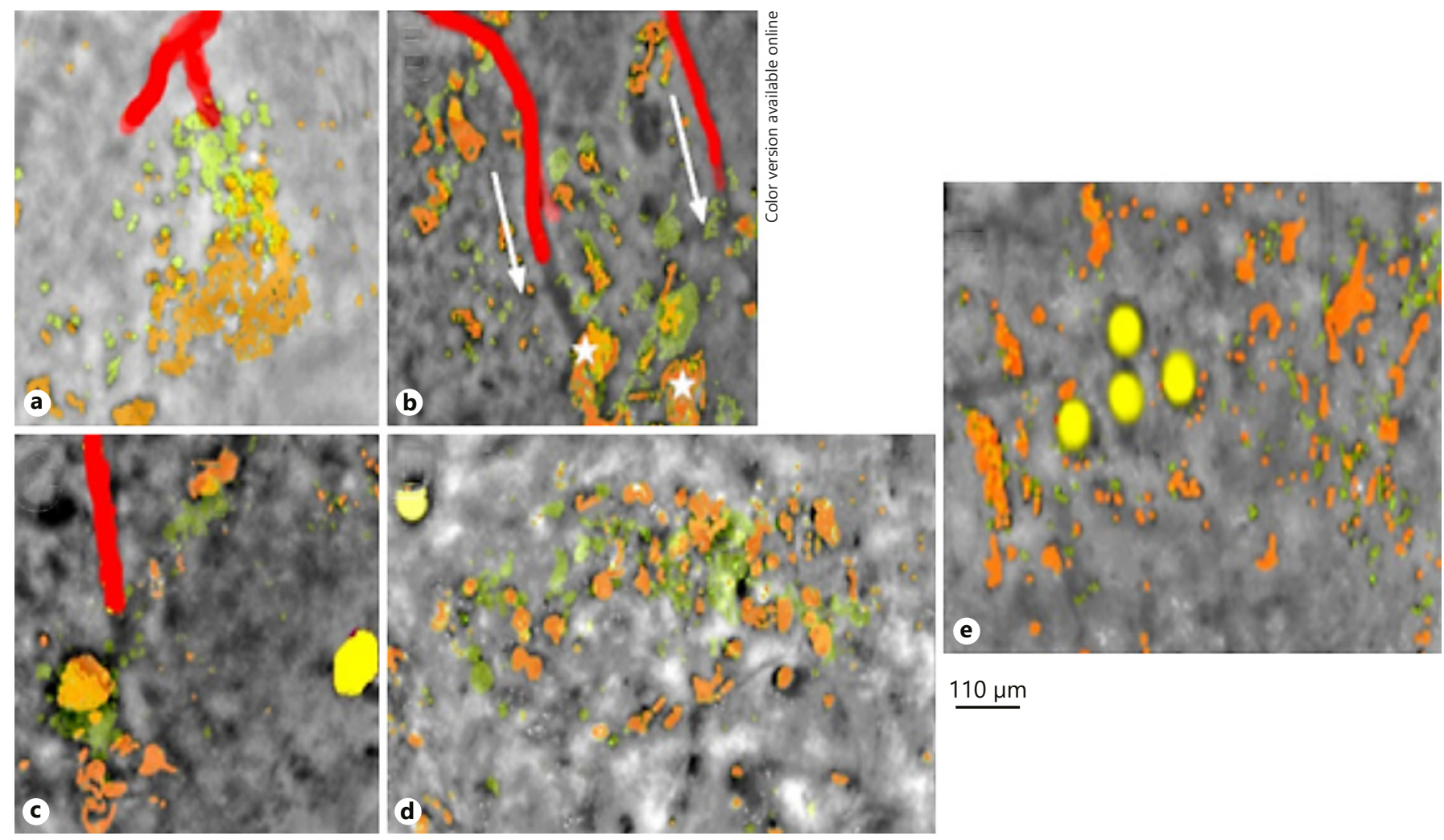

$110 \mu \mathrm{m}$

Fig. 3. Summary of hard exudate (HE) modification during the time of the study. Green, baseline aspect; orange, aspect at week 8 . Vascular landmarks (vessels and microaneurysm) are superimposed. Foci in $\mathbf{a}$ and $\mathbf{b}$ tend to aggregate in larger exudates. There is also a vertical displacement of the HEs that are spaced from the retinal vessels (white arrows). c, d The HEs are disrupted in a multitude of foci. e Circinate formation. area increased from $9.714 \mu \mathrm{m}^{2}$ to $18.431 \mu \mathrm{m}^{2}$, while the CRT remained stable. The number of foci in the region of interest decreased from 76 to 47 foci at 2 months (Fig. 2b). In the third case, the HE surface increased progressively according to a circinate pattern centered on a retinal microaneurysm that can be clearly seen on AO (see online suppl. Material 3 and Fig. 3).

\section{Discussion}

AO longitudinal imaging revealed constant change in HE size, shape, and location during the study period. Some changes were noted as soon as after 1 week. The presence of intraretinal edema did not prevent the imaging of the exudates. Some HEs moved slowly during the study period with an estimated speed of $1.59 \mu \mathrm{m}$ per day (Fig. 3b). The number of free foci was inversely correlated to the exudate surface. Foci appeared to play a key role in the natural history of HEs in both formation and disag- gregation, acting like the bricks of a wall under construction or demolition depending on the phase of the process. The nature of microfoci and foci are not known with certitude. HE is thought to be composed of lipid and proteinaceous material, such as fibrinogen and albumin, which form primarily in the outer plexiform layer of the retina [5]. The size $(20-30 \mu \mathrm{m})$ and oval shape of the foci suggest that they may correspond to foam cells that are lipid-laden macrophages [21]. In the histological report [2], colocalization of macrophages and apolipoprotein-B was observed around the retinal vasculature, supporting the hypothesis that macrophages play a role in cholesterol removal [21]. In the present study, foci were located in the neighborhood of vascular structures such as a retinal vessel and/or vessel abnormalities, as illustrated in case 3 where a circinate deposit made of an aggregation of foci appeared around a microaneurysm (see online suppl. Material 3). Bek [19] was the first to report the use of AO to image HE in diabetic retinopathy. Yamaguchi et al. [20] have recently reported two types of exudates using 
AO SLO in diabetic maculopathy that could not be distinguished using color fundus photography or SD-OCT: a round type consisting of an accumulation of spherical particles and an irregular type. Nevertheless, the aspect of HE in AOSLO images differed significantly from the images obtained with the camera used in the current study. In the current study, we also did not find a clear difference in exudate shape. In fact, it appeared that there was a kind of continuum between microfoci, foci, and the clinically visible exudates during HE formation.

This study has limitations such as the small number of eyes studied. However, the semiquantitative analysis performed identified rapid changes in the natural course of HE that were not reported previously. The area analyzed was also limited to the neighborhood of the clinically visible exudates located close to the fovea for evident practical reasons. It is not known if exudates farther from the center evolve in the same way. The camera used did not have an eye tracker guaranteeing that the same area was imaged over time. However, the good superposition of vascular landmarks during the study period strengthens the accuracy of the results presented. It should also be noted that in the absence of histological and clinical comparison, the interpretation of the $\mathrm{AO}$ images remains speculative.

In conclusion, $\mathrm{AO}$ made it possible to monitor rapid changes in HE progression. Surprisingly, a high turnover in a short period of time was observed. This method may contribute to a better understanding of the natural history of HEs and the efficacy of the various treatments available.

\section{Statement of Ethics}

The local ethics committee of the Besançon University Hospital approved the protocol and it adhered to the tenets of the Declaration of Helsinki. The nature of the study and its possible consequences were explained to study candidates, after which written informed consent was obtained from all participants.

\section{Disclosure Statement}

The authors have no conflicts of interest.

\section{Funding Sources}

The study did not receive any funding.

\section{Author Contributions}

Prisca Loganadane made substantial contributions to the conception of the work, to the acquisition, analysis, and interpretation of data for the work, and participated in drafting the work.

Bernard Delbosc approved the final version to be published and agreed to be accountable for all aspects of the work in ensuring that questions related to the accuracy or integrity of any part of the work are appropriately investigated and resolved.

Maher Saleh made substantial contributions to the conception of the work, participated in drafting the work and revising it critically for important intellectual content, approved the final version to be published, and agreed to be accountable for all aspects of the work in ensuring that questions related to the accuracy or integrity of any part of the work are appropriately investigated and resolved.

\section{References}

1 Chew EY, Klein ML, Ferris FL, Remaley NA, Murphy RP, Chantry K, et al. Association of elevated serum lipid levels with retinal hard exudate in diabetic retinopathy. Early Treatment Diabetic Retinopathy Study (ETDRS) Report 22. Arch Ophthalmol. 1996 Sep; 114(9):1079-84.

2 Cusick M, Chew EY, Chan CC, Kruth HS, Murphy RP, Ferris FL 3rd. Histopathology and regression of retinal hard exudates in diabetic retinopathy after reduction of elevated serum lipid levels. Ophthalmology. $2003 \mathrm{Nov}$; 110(11):2126-33

3 Sasaki M, Kawasaki R, Noonan JE, Wong TY, Lamoureux E, Wang JJ. Quantitative measurement of hard exudates in patients with diabetes and their associations with serum lipid levels. Invest Ophthalmol Vis Sci. 2013 Aug;54(8):5544-50.
4 Møller F, Bek T. The relation between visual acuity, fixation stability, and the size and location of foveal hard exudates after photocoagulation for diabetic maculopathy: a 1-year follow-up study. Graefes Arch Clin Exp Ophthalmol. 2003 Jun;241(6):458-62.

5 Holm K, Ponjavic V, Lövestam-Adrian M. Using multifocal electroretinography hard exudates affect macular function in eyes with diabetic retinopathy. Graefes Arch Clin Exp Ophthalmol. 2010 Sep;248(9):1241-7.

6 Otani T, Kishi S. Tomographic findings of foveal hard exudates in diabetic macular edema. Am J Ophthalmol. 2001 Jan;131(1):50-4.

7 Osareh A, Mirmehdi M, Thomas B, Markham R. Automated identification of diabetic retinal exudates in digital colour images. $\mathrm{Br} \mathrm{J}$ Ophthalmol. 2003 Oct;87(10):1220-3.
8 Ward NP, Tomlinson S, Taylor CJ. Image analysis of fundus photographs. The detection and measurement of exudates associated with diabetic retinopathy. Ophthalmology. 1989 Jan;96(1):80-6.

9 Bolz M, Schmidt-Erfurth U, Deak G, Mylonas G, Kriechbaum K, Scholda C; Diabetic Retinopathy Research Group Vienna. Optical coherence tomographic hyperreflective foci: a morphologic sign of lipid extravasation in diabetic macular edema. Ophthalmology. 2009 May;116(5):914-20.

10 Lammer J, Bolz M, Baumann B, Pircher M, Gerendas B, Schlanitz F, et al. Detection and analysis of hard exudates by polarization-sensitive optical coherence tomography in patients with diabetic maculopathy. Invest Ophthalmol Vis Sci. 2014 Mar;55(3):1564-71. 
11 Kim Y, Yu SY, Kwak HW. En Face SpectralDomain Optical Coherence Tomography Imaging of Outer Retinal Hard Exudates in Diabetic Macular Edema Based on Optical Coherence Tomography Patterns. Ophthalmic Surg Lasers Imaging Retina. 2016 Apr;47(4):313-21.

12 Takagi H, Otani A, Kiryu J, Ogura Y. New surgical approach for removing massive foveal hard exudates in diabetic macular edema. Ophthalmology. 1999 Feb;106(2):249-56.

13 Larsson J, Kifley A, Zhu M, Wang JJ, Mitchell P, Sutter FK, et al. Rapid reduction of hard exudates in eyes with diabetic retinopathy after intravitreal triamcinolone: data from a randomized, placebo-controlled, clinical trial. Acta Ophthalmol. 2009 May;87(3):275-80.
14 Pemp B, Deák G, Prager S, Mitsch C, Lammer J, Schmidinger G, et al.; Diabetic Retinopathy Research Group Vienna. Distribution of intraretinal exudates in diabetic macular edema during anti-vascular endothelial growth factor therapy observed by spectral domain optical coherence tomography and fundus photography. Retina. 2014 Dec;34(12):2407-15.

15 Domalpally A, Ip MS, Ehrlich JS. Effects of intravitreal ranibizumab on retinal hard exudate in diabetic macular edema: findings from the RIDE and RISE phase III clinical trials. Ophthalmology. 2015 Apr;122(4):779-86.

16 Gocho K, Sarda V, Falah S, Sahel JA, Sennlaub F, Benchaboune M, et al. Adaptive optics imaging of geographic atrophy. Invest Ophthalmol Vis Sci. 2013 May;54(5):3673-80.

17 Lombardo M, Parravano M, Serrao S, Ducoli P, Stirpe M, Lombardo G. Analysis of retinal capillaries in patients with type 1 diabetes and nonproliferative diabetic retinopathy using adaptive optics imaging. Retina. 2013 Sep; 33(8):1630-9.
18 Saleh M, Debellemanière G, Meillat $M$, Tumahai P, Bidaut Garnier M, Flores M, et al. Quantification of cone loss after surgery for retinal detachment involving the macula using adaptive optics. Br J Ophthalmol. 2014 Oct;98(10):1343-8.

19 Bek T. Fine structure in diabetic retinopathy lesions as observed by adaptive optics imaging. A qualitative study. Acta Ophthalmol. 2014 Dec;92(8):753-8.

20 Yamaguchi M, Nakao S, Kaizu Y, Kobayashi Y, Nakama T, Arima M, et al. High-Resolution Imaging by Adaptive Optics Scanning Laser Ophthalmoscopy Reveals Two Morphologically Distinct Types of Retinal Hard Exudates. Sci Rep. 2016 Sep;6(1):33574.

21 Kruth HS. Macrophage foam cells and atherosclerosis. Front Biosci. 2001 Mar;6(3):D429-55. 